\title{
Osteosarcoma arising from the parapharyngeal space: A case report
}

\author{
MANABU HOSHI, JUN TAKADA, NAOTO OEBISU and HIROAKI NAKAMURA \\ Department of Orthopedic Surgery, Osaka City University Graduate School of Medicine, Osaka 545-8585, Japan
}

Received January 16, 2014; Accepted June 5, 2014

DOI: $10.3892 / 01.2014 .2265$

\begin{abstract}
The current study presents a rare case of osteoblastic osteosarcoma arising from an extremely rare site, namely, the parapharyngeal space. To the best of our knowledge, this is the first study of computed tomography (CT) and magnetic resonance imaging (MRI) of an osteosarcoma in the parapharyngeal space with pathological correlation. A 56-year-old male presented with a mass of the right facial region. CT and MRI showed a heterogeneous mass, with ossification or calcification, occupying the parapharyngeal space. Open biopsy revealed an osteoblastic osteosarcoma containing calcified malignant osteoid. Lung CT also showed multiple lung metastases at the time of the first visit to the Department of Orthopedic Surgery, Osaka City University Graduate School of Medicine (Osaka, Japan). Systemic chemotherapy and radiotherapy were administered to the patient for palliation. The patient was alive at the 24-month follow-up subsequent to this treatment. Although a definitive diagnosis requires the use of a biopsy, the CT and MRI findings described in the present study suggest inclusion of this rare tumor in the differential diagnosis that is formed when such findings occur in the parapharyngeal space. The present study also briefly discusses osteosarcoma of the parapharyngeal space.
\end{abstract}

\section{Introduction}

Osteosarcoma is rare in the head and neck, accounting for $<0.5 \%$ (1) of all malignancies of this type. The majority of osteosarcomas in the head and neck are reported to occur in the mandible or maxilla, and few (10\%) occur in the skull and facial bones (2). Osteosarcoma arising in the parapharyngeal space is extremely rare. The current study presents this

Correspondence to: Dr Manabu Hoshi, Department of Orthopedic Surgery, Osaka City University Graduate School of Medicine, 1-4-3 Asahi-Machi, Abeno-ku, Osaka 545-8585, Japan

E-mail: hoshi@med.osaka-cu.ac.jp

Key words: osteosarcoma, parapharyngeal space, head and neck unusual case due to the rarity of the site, and subsequently discusses osteosarcoma of the parapharyngeal space.

\section{Case report}

A 56-year-old male presented with a mass in the right facial bone that had been apparent for three months, and dysesthesia, which had occurred gradually at this site. The patient incidentally identified the tumor and visited Wakakusa-Daiichi Hospital, Higashi-Osaka (Osaka, Japan), where the mass was confirmed with computed tomography (CT) examination. Nothing of note was found in the patient's past medical and family histories, and the findings from the routine laboratory studies were within the normal limits. Plain X-rays showed no particular findings, but regional CT showed a soft-tissue mass with prominent ossification in the central region, and involving the parapharyngeal space (Fig. 1), in which the anterior septum ballooned owing to compression from the mass. Magnetic resonance imaging (MRI) revealed heterogeneously low intensity to isointensity results on T1-weighted sequences and low to moderately high intensity results on T2-weighted images, with a size of $5.6 \times 4.8 \mathrm{~cm}$ (Fig. 2). Lung CT revealed multiple small nodules that were suspected to be lung metastases (Fig. 3).

An open biopsy was performed, and histologically, the lesion showed atypical cell proliferation with production of calcified malignant osteoid. The pathological diagnosis was of an osteoblastic osteosarcoma (Fig. 4). The disease was classified as advanced-stage IVB. At first, systemic chemotherapy was started with caffeine-assisted systemic high-dose chemotherapy, consisting of $60 \mathrm{mg} / \mathrm{m}^{2}$ Adriamycin and $120 \mathrm{mg} / \mathrm{m}^{2}$ cisplatin. However, subsequent to two courses of the regimen, a perforation of the sigmoid colon was found, and surgical repair was immediately performed with a colostomy. Moreover, irreversible renal dysfunction occurred, and this was worsened by sepsis following surgery. No discernable effect was observed from the chemotherapy on the mass in the parapharyngeal space and the lung target lesion. High-dose chemotherapy was deemed to be unsuitable for treating conventional osteosarcoma owing to the adverse effects. Thus, local radiation therapy, with a total dose of 39 Gy, and a total of seven courses of low-dose systemic chemotherapy (50-100 $\mathrm{mg} / \mathrm{m}^{2}$ cyclophospamide per day) for palliation, were administered. At the last follow-up, 24 months after the first visit, the size of the mass occupying the parapharyngeal space was unchanged, but the size and number of lung metastases 

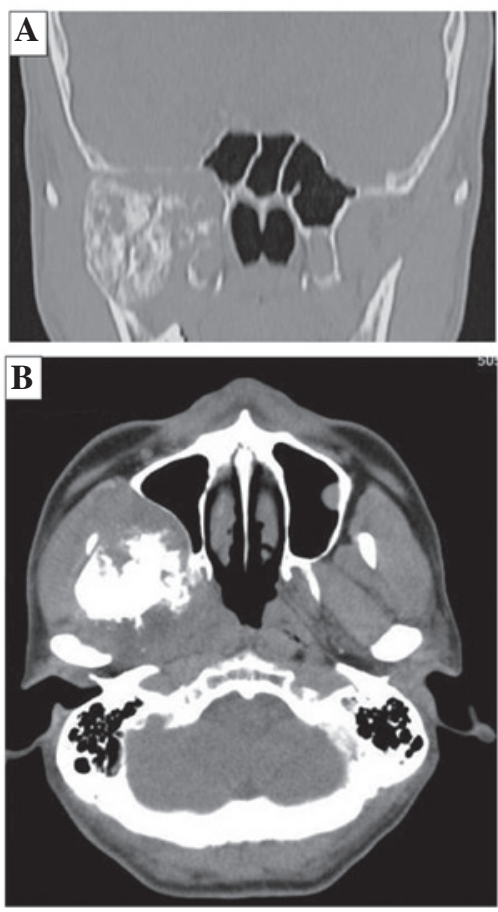

Figure 1. (A) Coronal and (B) axial view of regional computed tomography showing the mass with prominent ossification in the right parapharyngeal space.
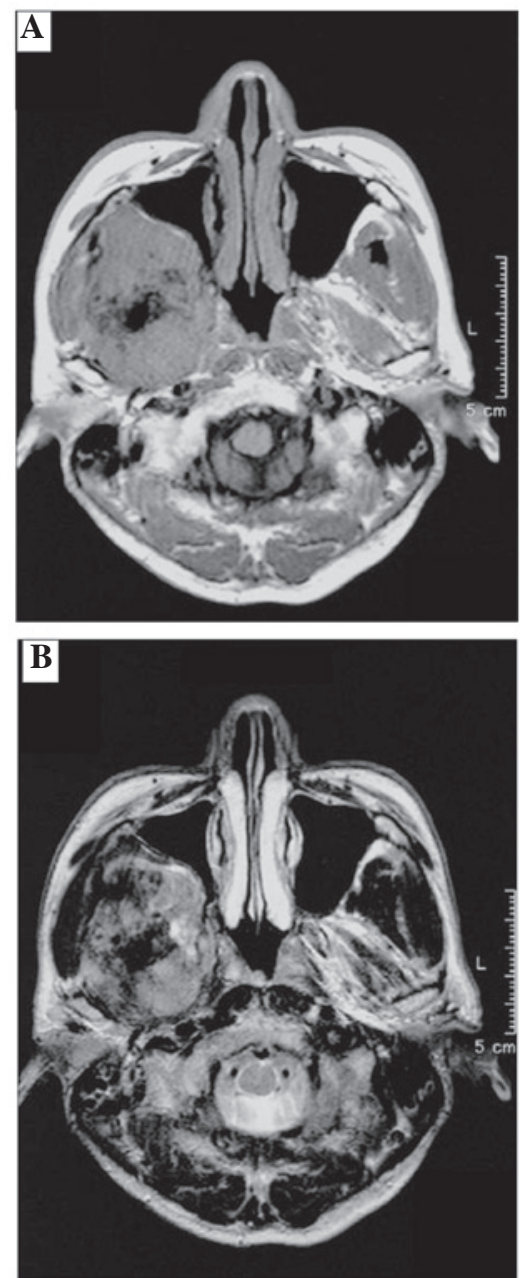

Figure 2. Low-intensity within an isointensity mass on an axial view of magnetic resonance imaging showing ossification in the tumor on (A) T1- and (B) T2-weighted images

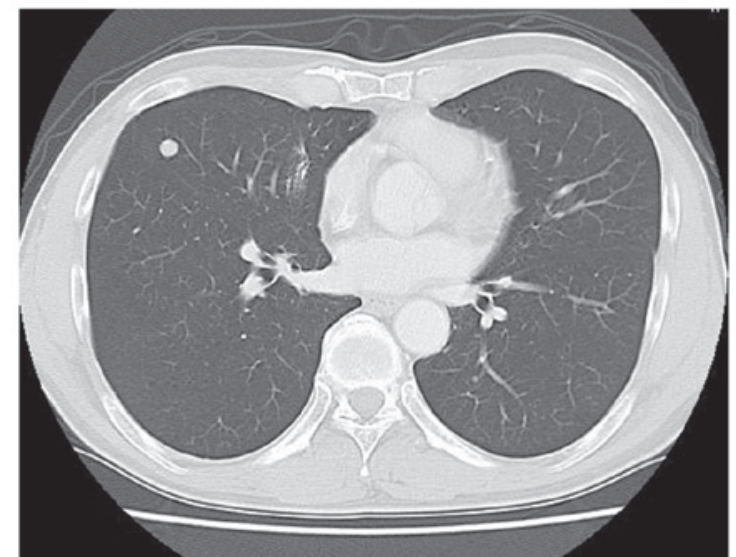

Figure 3. Lung computed tomography showing small nodules of lung metastases.

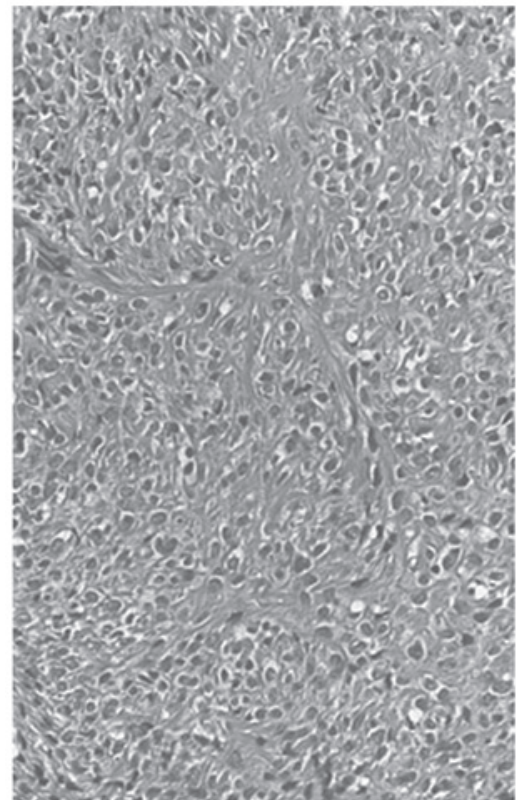

Figure 4. Biopsy specimen confirming osteoblastic osteosarcoma in which atypical cells produce calcified malignant osteoid (stain, hematoxylin and eosin; magnification, x200).

had increased. At the time of writing this study, the patient was alive with the disease. Written informed consent was obtained from the patient for publication of this case study and the accompanying images.

\section{Discussion}

Osteosarcoma is a primary high-grade malignant tumor in which the neoplastic cells produce osteoid (3). Osteosarcoma affects males more frequently than females. Young adolescents and adults under the age of 25 years are predominantly affected. Osteosarcoma usually arises in the bones around the knee, humeral joint and pelvis (4). The five-year survival rate is generally estimated to be $60-80 \%$ (3). However, the incidence of osteosarcoma in the head and neck regions is extremely low, and this form is reported to occur in adults in their $30 \mathrm{~s}$ and older (5). Cranial facial lesions account for $<10 \%$ of the total 
cases of osteosarcoma; these occur in the mandible and maxillary bones in particular. However it is difficult to find studies on osteosarcoma involving the parapharyngeal space (6).

Radiologically, tumor matrix mineralization and aggressive bone destruction are strongly suggestive of osteosarcoma. The present case demonstrated a high-density mass in the central area of the tumor on CT examination. On MRI, an intermediate intensity region was observed T1-weighted images and a heterogeneously high intensity region was observed on T2-weighted images. An ossified region in the central lesion, which was produced by tumor cells, showed a low intensity on each of the T1- and T2-weighted images. There was no discernible bone-forming reaction to indicate an osteosarcoma (7).

Histologically, osteosarcoma is an osteoid-producing tumor, and the identification of anaplastic stromal cells and the osteoid they produce aid in the histological diagnosis. The incidence of the classic type of osteosarcoma presents as osteoblastic $(50 \%)$, chondroblastic $(25 \%)$, or fibroblastic (25\%) in the extremities (3). Among head and neck osteosarcomas, chondroblastic osteosarcoma is the most common histopathological variant (8), while osteoblastic osteosarcoma is associated with a poor prognosis, differing from the chondroblastic type in the extremities (5).

Osteosarcoma usually presents with an aggressive course, with a high rate of distant metastasis and recurrence. In the absence of distant metastasis, the major therapeutic approach is surgery to achieve local control for high-grade osteosarcoma; surgery with a negative margin is necessary (9), and multimodality management, in addition to chemotherapy (10) and radiotherapy (11), is preferable. The anatomical site of the parapharyngeal space occupied by the tumor is adjacent to more critical structures in the head and neck, and radical surgery would have great disadvantages in terms of function and possible cosmetic problems. However, systemic chemotherapy with high-dose treatment may be the main treatment for cases with distant metastases. The adverse effect of irreversible renal dysfunction emerged in the present case following the two initial courses of chemotherapy, and high-dose chemotherapy could not be recommended.

The five-year survival rate of patients with head and neck osteosarcoma is estimated to be $57-63 \%(2,11)$, which is lower than the $70-80 \%$ survival rate of patients with osteosarcoma of the extremities (12). Regarding prognostic factors, Smith et al (5) proposed that a poor prognosis is associated with an age of $>60$ years, a non-mandibular tumor location, a tumor size of $>6 \mathrm{~cm}$, an osteoblastic histological type, an advanced disease stage, non-surgical initial therapy and a positive margin of resection. At the latest follow-up, the present patient was alive with the disease, but unfortunately possessed a number of these poor prognostic factors.

In conclusion, the present study presents a rare case of osteoblastic osteosarcoma arising from a rare lesion of the parapharyngeal space. Although a definitive diagnosis requires a biopsy, the CT and MRI findings described in this study suggest the inclusion of this rare tumor in the differential diagnosis when such findings occur in the parapharyngeal space.

\section{References}

1. de Fries HO, Perlin E and Leibel SA: Treatment of osteogenic sarcoma of the mandible. Arch Otolaryngol 105: 358-359, 1979.

2. Canadian Society of Otolaryngology-Head and Neck Surgery Oncology Study Group: Osteogenic sarcoma of the mandible and maxilla: a Canadian review (1980-2000). J Otolaryngol 33: 139-144, 2004

3. Raymond AK, Ayala AG and Knuuutila S: Conventional osteosacoma. In: Fletcher CDM, Unni KK and Mertens F (eds). World Health Organization Classification of Tumors: Pathology and Genetics; Tumors of Soft Tissue and Bone. Vol. 4. IARC Press, Lyon, pp264-270, 2002.

4. Unni KK, Inwards CY, Kindblom LG and Wold LE (eds): Osteosarcoma of bone. In: Tumors of the Bones and Joints. umors of the bones and joints. AFIP atlas of tumor pathology, series 4 . American Registry of Pathology, Washington DC, pp135-170, 2005.

5. Smith RB, Apostolakis LW, Karnell LH, Koch BB, Robinson RA, Zhen W, Menck HR and Hoffman HT: National Cancer Data Base report on osteosarcoma of the head and neck. Cancer 98: 1670-1680, 2003.

6. Dorfman HD and Czerniak B (eds): Osteosarcoma. In: Bone Tumors. Mosby, Inc, St. Louis, pp128-253, 1998.

7. Park HR, Min SK, Cho HD, Cho SJ, Lee JH, Lee Y and Park YK: Osteosarcoma of the ethmoid sinus. Skeletal Radiol 33: 291-294, 2004.

8. Laskar S, Basu A, Muckaden MA, D'Cruz A, Pai S, Jambhekar N, Tike P and Shrivastava SK: Osteosarcoma of the head and neck region: lessons learned from a single-institution experience of 50 patients. Head Neck 30: 1020-1026, 2008.

9. Patel SG, Meyers P, Huvos AG, Wolden S, Singh B, Shaha AR, Boyle JO, Pfister D, Shah JP and Kraus DH: Improved outcomes in patients with osteogenic sarcoma of the head and neck. Cancer 95: 1495-1503, 2002

10. Tsuchiya H, Tomita K, Mori Y, Asada N and Yamamoto N: Marginal excision for osteosarcoma with caffeine assisted chemotherapy. Clin Orthop Relat Res 358: 27-35, 1999.

11. Guadagnolo BA, Zagars GK, Raymond AK, Benjamin RS and Sturgis EM: Osteosarcoma of the jaw/craniofacial region: outcomes after multimodality treatment. Cancer 115: 3262-3270, 2009.

12. Kawaguchi N, Ahmed AR, Matsumoto S, Manabe J and Matsushita Y: The concept of curative margin in surgery for bone and soft tissue sarcoma. Clin Orthop Relat Res 419: 165-172, 2004. 